\title{
AVALIAÇÃO GENÉTICA DE SELEÇÕES E HÍBRIDOS DE LIMÕES CRAVO, VOLKAMERIANO E RUGOSO COMO PORTA-ENXERTOS PARA LARANJEIRAS VALÊNCIA NA PRESENÇA DA MORTE SÚBITA DOS CITROS ${ }^{1}$
}

\author{
JORGINO POMPEU JUNIOR ${ }^{2}$, SILVIA BLUMER ${ }^{3}$, MARCOS DEON VILELA DE RESENDE ${ }^{4}$
}

RESUMO - Este estudo teve como objetivo realizar a avaliação genética da produção de frutos, eficiência produtiva e altura de laranjeiras Valência (Citrus sinensis) enxertadas em seleções e híbridos dos limões Cravo (C. limonia), Volkameriano (C. volkameriana) e Rugoso (C. jambhiri), em área endêmica para morte súbita dos citros (MSC). Foram avaliados 36 genótipos desses porta-enxertos, representados por cinco plantas cada, avaliados em cinco safras, do terceiro ao sétimo ano após o plantio. Sete dos genótipos avaliados apresentaram plantas com sintomas de MSC até o sétimo ano: Rangpur Otaheite orange 12901 (859), Rangpur Red Lime D.33.30 (866), Limão-Cravo EEL (871), Rangpur Borneo red (874), Citrus kokhai (1649), Limão-Rugoso 58329 (1655) e Limão- Cravo x Swingle B (1695). Para os genótipos que não manifestaram sintomas da doença, foram estimados parâmetros genéticos e fenotípicos e realizada a predição de valores genéticos dos indivíduos, visando à seleção e ao melhoramento genético para as características citadas, empregando-se o método REML/BLUP (máxima verossimilhança restrita/melhor predição linear não viciada). A análise de produção de frutos de cinco safras mostrou acurácia seletiva de 84,59\%, tornando-se desnecessária a avaliação de maior número de safras. A seleção dos sete melhores genótipos proporcionou ganhos genéticos de 11,5\% na produção de frutos, enquanto a do melhor genótipo conferiu ganho genético de 16,3\%. As maiores médias genéticas preditas $\left(>70,0 \mathrm{~kg} \cdot \mathrm{pl}^{-1}\right)$ para produção de frutos foram obtidas pelos genótipos Limão-Cravo- Ipanema (1522), Santa- Bárbara-Red- Lime (884), Limão- Cravo- Limeira (863), Limão- Cravo- Taquaritinga (869), Limão- Rugoso- do -Cabo (1643), Rangpur- Rose Lime (868) e Limão- Cravo- da- Califórnia (1467). Já a acurácia seletiva da eficiência produtiva, para quatro colheitas, foi 77,4\%. Para este caráter, as maiores médias genéticas $\left(>8,0 \mathrm{~kg} \cdot \mathrm{m}^{-3}\right)$ foram dos genótipos RangpurLime x Trifoliata 3810 (1648), Rangpur- Lime x Trifoliata 5320 (1644), Limão- Cravo x Citrange- Carrizo (1524), Citrus pennivesiculata (880), Limão- Cravo x Trifoliata- Swingle A (1707), Rangpur- Rose- Lemon 124684 (864), Rangpur- Red -Lime D33.47 (867) e Limão- Cravo -Ipanema (1522). Dentre os 10 melhores genótipos para produção de frutos e para eficiência produtiva, apenas três são coincidentes: Rangpur- Rose -Lime (868), Citrus pennivesiculata (880) e Limão- Cravo-Ipanema (1522).

Termos para indexação: melhoramento, parâmetros genéticos, REML/BLUP, Morte Súbita dos Citros, Citrus limonia, Citrus volkameriana, Citrus jambhiri.

\section{GENETIC EVALUATION OF SELECTIONS AND HYBRIDS OF RANGPUR LIME, VOLKAMER AND ROUGH LEMONS ROOTSTOCKS FOR VALENCIA ORANGE TREES IN THE PRESENCE OF THE CITRUS SUDDEN DEATH}

ABSTRACT - This study aimed to perform the genetic evaluation of fruit production, productive efficiency and growth of Valência orange (Citrus sinensis) trees scions grafted on selections and hybrids of Rangpur lime (C. limonia), Volkamer (C. volkameriana) and Rough (C. jambhiri) lemons rootstocks grown in the presence of the Citrus Sudden Death (CSD). In an affected endemic area for CSD 36 genotypes of these rootstocks were evaluated, represented by five plants each one, measured in five harvests from the third to seventh years after planting. Seven of them showed symptoms of CSD: Rangpur Otaheite orange 12901 (859), Rangpur Red Lime D.33.30 (866), Limão Cravo EEL (871), Rangpur Borneo red (874), Citrus kokhai (1649), Rough lemon 58329 (1655) and Limão Cravo x Swingle B (1695). For selection and breeding purposes, genetic and phenotypic parameters were estimated and individual genotypic values were predicted for all traits by the REML/BLUP (restricted maximum likelihood/best linear unbiased prediction) procedure. The analysis of fruit production in five harvests showed selective accuracy of $84.59 \%$ revealing that a greater number of harvests is unnecessary. Selection of the best seven genotypes led to a genetic gain of $11.5 \%$ for fruit production while the selection of the very best genotype provided an estimated genetic gain of $16.3 \%$. The higher predicted genetic means $(>70.0$ kg. $\mathrm{p}^{-1}$ ) for fruit production were obtained for the genotypes Ipanema Rangpur Lime (1522), Santa Bárbara Red Lime (884), Limeira Rangpur Lime (863), Taquaritinga Rangpur Lime (869), Do Cabo Rough Lemon (1643), Rangpur Rose Lime (868) and Califórnia Rangpur Lime (1467). The selective accuracy for the productive efficiency based on four harvests was $77.4 \%$. For this trait the higher predicted genetic means $\left(>8.0 \mathrm{~kg}^{\mathrm{p} \mathrm{p}^{-1}}\right)$ were obtained for the genotypes Rangpur Lime x Trifoliata 3810 (1648), Rangpur Lime x Trifoliata 5320 (1644), Rangpur Lime x Citrange Carrizo (1524), Citrus pennivesiculata (880), Rangpur Lime x Trifoliata Swingle A(1707), Rangpur Rose Lemon 124684 (864), Rangpur Red Lime D33.47 (867) and Ipanema Rangpur Lime (1522). Among the best 10 genotypes for fruit production and for productive efficiency only three are coincident: Rangpur Rose Lime (868), Citrus pennivesiculata (880) and Ipanema Rangpur Lime (1522).

Index terms: breeding, quantitative genetics, REML/BLUP, Citrus Sudden Death, Citrus limonia, Citrus volkameriana, Citrus jambhiri.

${ }^{1}$ (Trabalho 094-12). Recebido em: 08-02-2012. Aceito para publicação em: 14-02-2013.

${ }^{2}$ Centro APTA Citros Sylvio Moreira, Cordeiropolis-SP. Bolsista 2 CNPq. E-mail: jorgino@centrodecitricultura.br

${ }^{3}$ Pós Doutoranda em Fitopatologia, Esalq/USP, Lab. de Fisiologia e Bioq. Fitopatológica, Bolsista CNPq/PNPD. Email: silviablumer@yahoo.com.br ${ }^{4}$ Embrapa Florestas/Universidade Federal de Viçosa, Viçosa, MG, Brasil. E-mail: deon@enpf.embrapa.br 


\section{INTRODUÇÃO}

Desde 1999, a morte súbita dos citros (MSC) vem afetando laranjeiras (Citrus sinensis L. Osb.) e outras espécies cítricas enxertadas nos limões Cravo (C. limonia L. Osb.), Volkameriano (C. volkameriana V. Tenn. et Pasq.) e Rugoso (C. jambhiri Lush.), em Minas Gerais e São Paulo. Inicialmente, as folhas das plantas afetadas perdem o brilho, adquirem coloração verde-pálida, seguindo-se a queda das folhas e a morte dos ramos apicais, culminando com o perecimento das plantas, de poucas semanas a dois anos após o aparecimento dos sintomas. A retirada da casca dos porta-enxertos intolerantes revela o amarelecimento na região cambial, sendo esse o sintoma-diagnóstico da doença e que precede os sintomas da copa (BASSANEZI et al., 2003). A MSC já foi observada em plantas com dois ou mais anos de idade, sendo mais comum a partir do quinto ano. Plantas enxertadas sobre as tangerinas Cleópatra (C. reshni hort. ex Tan.) e Sunki [C. sunki (Hayata) hort. ex Tan.], trifoliata (Poncirus trifoliata Raf.) e citrumelo Swingle [C. paradisi Macf. x P. trifoliata Raf.] são assintomáticas, e estes porta-enxertos são considerados tolerantes à MSC. Entretanto, borbulhas retiradas da copa destas plantas e enxertadas em plantas com porta-enxertos intolerantes são capazes de induzir os sintomas da doença nestas plantas (YAMAMOTO et al., 2011). A inserção de interenxerto de trifoliata ou de tangerina Cleópatra entre o limoeiro Cravo e a laranjeira Valência (C. sinensis L. Osb.) não impediu a manifestação da doença (POMPEU JÚNIOR; BLUMER, 2008). Desconhece-se o agente causal da MSC supondo-se ser um vírus transmitido por vetor alado e por borbulha coletada de plantas sintomáticas e assintomáticas (BASSANEZI et al., 2003; ROMAN et al., 2004; MACCHERONI et al., 2005; YAMAMOTO et al., 2011).

A convivência com a MSC vem sendo feita com a utilização dos porta-enxertos tolerantes, todos mais suscetíveis à seca que os limões Cravo, Volkameriano e Rugoso.

A existência de seleções e híbridos desses porta-enxertos no Banco Ativo de Germoplasma de Citros do Centro APTA Citros Sylvio Moreira, do Instituto Agronômico (BAG do CAPTA Citros/IAC) e em outras coleções no Brasil e no exterior, motivou a introdução e a avaliação desse germoplasma na região de ocorrência da MSC.

A avaliação de materiais genéticos em experimentos de campo tem dois objetivos: (i) inferir sobre os valores genotípicos de tais materiais e (ii) ordenar os materiais genéticos com base em seus valores genotípicos. Não há interesse em estimar as médias fenotípicas dos materiais genéticos nos experimentos e, sim, estimar suas médias genéticas (ou valores genotípicos), ou seja, suas médias futuras, quando forem plantados novamente em plantios comerciais. Quando plantados comercialmente, mesmo que seja no mesmo local ou região da experimentação, os efeitos de blocos, parcelas e efeitos ambientais aleatórios não se repetirão. Como tais efeitos estão embutidos em alguma proporção nas médias fenotípicas, isto prova que tais médias não são adequadas para inferência sobre os valores genotípicos dos materiais genéticos (RESENDE; DUARTE, 2007).

A avaliação genotípica em plantas compreende a estimação de componentes de variância (parâmetros genéticos) e a predição dos valores genotípicos. Além da utilidade no processo de predição dos valores genéticos, as estimativas dos parâmetros genéticos, tais quais a herdabilidade e a repetibilidade, são fundamentais para o delineamento de eficientes estratégias de melhoramento e seleção. O procedimento ótimo de avaliação genotípica refere-se ao REML/BLUP (máxima verossimilhança residual ou restrita/melhor predição linear não viciada), também denominado genericamente de metodologia de modelos mistos (RESENDE, 2007). Esse método produz o ordenamento genético dos materiais em avaliação, eliminando todas as influências ambientais incidentes sobre os fenótipos medidos.

Este estudo teve como objetivo realizar a avaliação genética da produção de frutos, eficiência produtiva e altura de laranjeiras Valência enxertadas em seleções e híbridos dos limões Cravo, Volkameriano e Rugoso, em condições de sequeiro, em região de ocorrência da MSC.

\section{MATERIAL E MÉTODOS}

O ensaio foi instalado em maio de 2003, em Comendador Gomes-MG, (coordenadas 1942'46" sul e 48 57'58" oeste) sob clima Cwa (SETZER, 1966) em Latosol Vermelho-Amarelo, em área anteriormente ocupada por pomares erradicados pela presença da MSC e vizinho de pomares portadores da doença, no espaçamento 7,5 m x 3,5 m e conduzido sem irrigação. Foram avaliados os porta-enxertos relacionados na Tabela 1 com seus números de registro no BAG do CAPTA Citros/IAC

Como copa, foi selecionada a laranja Valência por ser suscetível à MSC quando enxertada nos limões Cravo e Volkameriano, ser a segunda cultivar-copa mais importante da citricultura paulista e ser compatível com a quase totalidade dos porta-enxertos, exceto o limão Cravo x citrange Carrizo [C. limonia $x$ (P.trifoliata $x$ C. sinensis)] (POMPEU 
JÚNIOR ; BLUMER, 2002, 2009).

Foram avaliados 36 genótipos de porta-enxertos, cada qual representado por cinco plantas enxertadas com laranja Valência plantadas lado a lado na mesma linha de plantio, sem delineamento experimental, visando a facilitar as comparações entre os porta-enxertos que mostrassem tolerância a MSC.

Os dados de produção de frutos foram obtidos em cinco safras anuais, no período de 2006-2010, e as medições da altura das plantas e do diâmetro das copas realizados anualmente, após as colheitas, de 2007 a 2010. A eficiência produtiva anual foi calculada com a fórmula $\mathrm{EP}=\mathrm{P} / 0,52 \mathrm{D}^{2} \mathrm{H}$, em que: $\mathrm{P}$ representa a produção de frutos; $\mathrm{D}$ o diâmetro da copa, e H a altura da planta (MENDEL, 1956).

As análises foram realizadas empregando-se a metodologia de modelos mistos, com estimação de componentes de variância, pelo método da máxima verossimilhança restrita (REML) e predição de valores genotípicos pela melhor predição linear não viciada (BLUP) (RESENDE, 2002). O seguinte modelo estatístico foi empregado: $\mathrm{y}=\mathrm{Xm}+\mathrm{Zg}+$ $\mathrm{Wp}+\mathrm{e}$, em que: y é o vetor de dados; $\mathrm{m}$ é o vetor dos efeitos de medição ou safra (assumidos como fixos), somados à média geral; g é o vetor dos efeitos genotípicos (assumidos como aleatórios); p é o vetor dos efeitos de ambiente permanente (assumidos como aleatórios); e é o vetor de erros ou resíduos (aleatórios). As letras maiúsculas, $\mathrm{X}, \mathrm{Z}$ e $\mathrm{W}$, representam as matrizes de incidência para os referidos efeitos. As distribuições e estruturas de médias e variâncias associadas ao modelo são:

$E\left[\begin{array}{l}y \\ g \\ p \\ e\end{array}\right\rfloor=\left[\begin{array}{c}\Delta / n \\ 0 \\ 0 \\ 0\end{array}\right] ; \quad \operatorname{Var}\left[\begin{array}{l}g \\ p \\ e\end{array}\right]=\left[\begin{array}{ccc}I \sigma_{z}^{2} & 0 & 0 \\ 0 & I \sigma_{p}^{2} & 0 \\ 0 & 0 & I \sigma_{s}^{2}\end{array}\right]$,

As equações de modelo misto para a predição BLUP são dadas por:

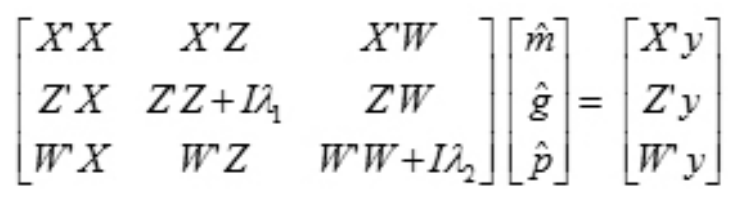

em que:

$\lambda_{1}=\frac{\sigma_{\varepsilon}^{2}}{\sigma_{g}^{2}}=\frac{1-h_{g}^{2}-p^{2}}{h_{g}^{2}} ; \quad \lambda_{2}=\frac{\sigma_{\varepsilon}^{2}}{\sigma_{p}^{2}}=\frac{1-h_{g}^{2}-p^{2}}{p^{2}}$. $h_{g}^{2}=\frac{\sigma_{g}^{2}}{\sigma_{g}^{2}+\sigma_{p}^{2}+\sigma_{\varepsilon}^{2}}=$ herdabilidade individual no sentido amplo ou proporção da variação total entre plantas no experimento, devida a causas genéticas; $p^{2}=\frac{\sigma_{p}^{2}}{\sigma_{g}^{2}+\sigma_{p}^{2}+\sigma_{\varepsilon}^{2}}=$ coeficiente de determinação dos efeitos de ambiente permanente;

$\sigma_{g}^{2}=$ variância genotípica, que mede a quantidade de variação no experimento, devida a causas genéticas;

$\sigma_{p}^{2}=$ variância dos efeitos de ambiente permanente, que mede a quantidade de variação no experimento, devida a efeitos de ambiente que são constantes através das safras.

$\sigma_{e}^{2}=$ variância residual ou devida a variações ambientais aleatórias entre os anos (por exemplo, variações associadas às mudanças climáticas entre anos);

$\sigma_{y}^{2}=\frac{\sigma_{p}^{2}}{\sigma_{g}^{2}+\sigma_{p}^{2}+\sigma_{\varepsilon}^{2}} \quad=$ variância fenotípica individual ou variação total entre plantas no experimento; $\rho=\frac{\sigma_{g}^{2}+\sigma_{p}^{2}}{\sigma_{g}^{2}+\sigma_{p}^{2}+\sigma_{\varepsilon}^{2}} \quad=$ repetibilidade individual ou correlação entre o comportamento dos fenótipos ao longo das safras.

Os estimadores de componentes de variância pelo método REML via algoritmo EM equivalem a:

$\hat{\sigma}_{c}^{2}=\left[\begin{array}{llll}y^{\prime} y-\hat{b}^{\prime} & \left.X^{\prime} y-\hat{g}^{\prime} \quad Z^{\prime} y-\hat{p}^{\prime} W^{\prime} y\right] /[N-r(x)]\end{array}\right.$

$\left.\hat{\sigma}_{\bar{g}}^{2}=\left[\hat{g}^{\prime} \hat{g}+\hat{\sigma}_{0}^{2} \operatorname{tr} C^{22}\right)\right] / q$

$\hat{\sigma}_{p}^{2}=\left[\hat{p}^{\prime} \hat{p}+\hat{\sigma}_{e}^{2} \operatorname{tr} C^{33}\right] / s$

em que $C^{22}$ e $C^{33}$ advêm de :

$$
\mathrm{C}^{-1}=\left[\begin{array}{lll}
C_{11} & C_{12} & C_{13} \\
C_{21} & C_{22} & C_{23} \\
C_{31} & C_{32} & C_{33}
\end{array}\right]^{-1}=\left[\begin{array}{lll}
C^{11} & C^{12} & C^{13} \\
C^{21} & C^{22} & C^{23} \\
C^{31} & C^{32} & C^{33}
\end{array}\right]
$$

$\mathrm{C}=$ matriz dos coeficientes das equações de modelo misto;

$\operatorname{tr}=$ operador-traço matricial;

$\mathrm{r}(\mathrm{x})=$ posto da matriz $\mathrm{X}$;

$\mathrm{N}, \mathrm{q}, \mathrm{s}=$ número total de dados, número de genótipos e número de indivíduos, respectivamente. 


$$
\text { O estimador } \hat{h}_{z}^{2}=\frac{\hat{\sigma}_{z}^{2}}{\hat{\sigma}_{z}^{2}+\hat{\sigma}_{\theta}^{2}+\hat{\sigma}_{c}^{2}} \text { foi empre- }
$$

gado para o cômputo da herdabilidade individual no sentido amplo, em que é a estimativa da variância genotípica, é a estimativa da variância de ambiente permanente entre plantas e é a estimativa da variância residual. O estimador $\hat{\rho}=\frac{\hat{\sigma}_{s}^{2}+\hat{\sigma}_{b}^{2}}{\hat{\sigma}_{z}^{2}+\hat{\sigma}_{p}^{2}+\hat{\sigma}_{c}^{2}}$ foi empreempregado para o cômputo da repetibilidade individual. As demais estimativas de herdabilidades, repetibilidades e acurácias foram obtidas via inversão da matriz dos coeficientes $\left(\mathrm{C}^{-1}\right)$, conforme descrito acima, das equações de modelo misto, conforme detalhado por Resende (2002). A seguir são apresentados os significados desses parâmetros:herdabilidade ao nível de médias de cinco plantas em uma safra: confiabilidade da seleção baseada na média de cinco plantas do genótipo em uma safra;

- herdabilidade ao nível de médias de cinco plantas em todas as safras: confiabilidade da seleção baseada na média de cinco plantas do genótipo em todas as safras;

- repetibilidade ao nível de médias de cinco safras: coeficiente de determinação dos fenótipos com base na média de todas as safras;

- acurácia na seleção de genótipos em uma safra: correlação entre o valor genético verdadeiro e aquele estimado com base na avaliação em uma só safra;

- acurácia na seleção de genótipos com base na média de todas as safras: correlação entre o valor genético verdadeiro e aquele estimado com base na avaliação em todas as safras.

A acurácia seletiva contempla também a magnitude relativa das variações genética e ambiental no experimento. A inferência a partir do experimento será considerada de elevada precisão se houver alta variabilidade genética entre os seus genótipos e baixa variabilidade ambiental. Nesse caso, há um alto poder discriminatório no experimento para o caráter que está sendo avaliado.

As correlações genéticas foram estimadas por meio da correlação de Pearson entre os valores genéticos preditos (via BLUP) dos genótipos para todas as variáveis. Distâncias Euclidianas médias entre os genótipos foram utilizadas para a análise de agrupamento pelo método mutuamente exclusivo de Tocher (RESENDE, 2007).

A distância euclidiana ou métrica euclidiana equivale à raiz quadrada do somatório da diferença quadrática entre os valores de cada variável observada em dois indivíduos ou pontos de um espaço v-dimensional, em que $\mathrm{v}$ é o número de variáveis ou coordenadas no espaço. Matematicamente, é dada por $D_{E}\left(i, i^{\prime}\right)=$ $\left.\left[\sum_{j=1}^{v}\left(u_{i j}-u_{i^{\prime} j}\right)^{2}\right)\right]^{1 / 2}$ em que i e $i^{\prime}$ referem-se aos dois indivíduos em questão e $u_{\mathrm{ij}}$ e $u_{\mathrm{i}^{\prime} \mathrm{j}}$ referem-se aos valores observados para a variável j nos indivíduos i e i'. Os valores obtidos por essa expressão são muito altos quando o número de variáveis é elevado. Em função disso, formas alternativas dessa distância são mais usadas na prática, tais quais a distância euclidiana média dada por $D_{E M}\left(i, i^{\prime}\right)=\left(\sum_{i=1}^{v}\left(u_{i j}-u_{i^{\prime} j}\right)^{2 / v}\right]^{1 / 2} \mathrm{e}$ o quadrado da distância euclidiana média dado por $D_{Q E M}\left(i, i^{\prime}\right)=\left(\sum_{j=1}^{v}\left(u_{i j}-u_{i^{\prime} j}\right)^{2}\right) / v$. A distância euclidiana não é invariante aos efeitos de escala de medição das variáveis. Assim, as variáveis usadas em $u_{i j}$ e $u_{i^{\prime} j}$ devem ser previamente padronizadas, dividindo-as por seus respectivos desvios-padrão.

Todas as análises foram realizadas por meio do software Selegen-Reml/Blup (RESENDE, 2007).

\section{RESULTADOS E DISCUSSÃO}

A primeira constatação da MSC no experimento foi em dezembro de 2006. No decorrer do experimento, mostraram sintomas da doença em duas ou mais plantas e foram excluídos da análise estatística os porta-enxertos: Rangpur-Otaheite orange 12901 (859), Rangpur-Red- Lime D.33.30 (866), Limão- Cravo- EEL (871), Rangpur- Borneo red (874), Citrus kokhai (1649), Limão- Rugoso 58329 (1655) e Limão- Cravo x Swingle B (1695).

A Tabela 2 apresenta os dados médios anuais de produção de frutos, as eficiências produtivas médias anuais e a altura média dos tratamentos em 2010. Na Tabela 3, são apresentadas as estimativas de parâmetros genéticos para a produção de frutos, altura da planta e eficiência produtiva de laranjeiras Valência sobre os 29 porta-enxertos que não manifestaram sintomas de MSC até o sétimo ano após o plantio.

Para a produção de frutos, a estimativa da herdabilidade individual no sentido amplo, associada a uma safra em uma planta, foi de 9,3\%. Essa baixa herdabilidade é típica de um caráter quantitativo governado por muitos genes e com elevada influência ambiental. Também há uma dificuldade prática de quantificar precisamente essa variável, enquanto outras variáveis como a altura permitem uma medição mais exata. Nesse caso, a seleção, para ser precisa, necessita de várias repetições de cada genótipo e a colheita de várias safras. A herdabilidade ao nível de médias de cinco plantas de um mesmo genótipo, em uma safra, elevou-se a 33,9\%, e a herdabilidade da média das cinco plantas nas cinco safras foi de $71,57 \%$. Esse valor é ótimo para a seleção, pois conduz a uma acurácia seletiva de 84,59\% (Tabela 3). No contexto da avaliação genotípica, o parâmetro estatístico mais importante é a acurácia seletiva. 
Esse parâmetro refere-se à correlação entre o valor genotípico verdadeiro do material genético e aquele estimado ou predito a partir das informações dos experimentos de campo. A acurácia é tanto mais alta quanto menores forem os desvios absolutos entre os valores genéticos paramétricos ou verdadeiros e os valores genéticos estimados ou preditos (RESENDE, 2007). A repetibilidade individual em uma safra foi baixa, em torno de $10 \%$. Mas ao nível de cinco safras, elevou-se a $35,15 \%$ (Tabela 3 ). A acurácia seletiva obtida foi de $84,59 \%$ e para obtenção de uma acurácia de $90 \%$ seriam necessárias nove safras, valor esse obtido com base na expressão matemática que extrapola os valores da acurácia em função do número de safras colhidas. Assim, as cinco safras empregadas, praticamente já maximizaram a acurácia seletiva.

As médias genéticas preditas para o caráter produção de frutos, bem como seus limites inferiores (LIIC) e superiores (LSIC) do intervalo de confiança a 95\% de confiança, são apresentadas na Tabela 4 . Verifica-se que a produção média por planta em uma safra variou de 52,45 a 75,48 , com média 64,92 . As médias genéticas dos melhores materiais apresentam menores valores do que suas médias fenotípicas, pois os efeitos ambientais foram eliminados no processo de predição.

Em trabalhos científicos e em catálogos de avaliação genética, a apresentação de médias fenotípicas não é desejável e recomendável. Pelo contrário, devem ser apresentados os valores genotípicos livres dos efeitos ambientais. Esses valores genotípicos são os verdadeiros valores de cultivo e uso (VCU). Atualmente, os testes de VCU têm sido requisitados pelo Ministério da Agricultura para fins legais de recomendação, registro e proteção de cultivares. É importante relatar que o conceito de VCU é exatamente o conceito de valores genotípicos e, portanto, o uso de médias fenotípicas para inferência em testes de VCU não é recomendável (RESENDE; DUARTE, 2007).

A seleção dos sete melhores porta-enxertos conduz a uma média da população melhorada de $72,42 \%$, produzindo um ganho genético de $11,55 \%$ na produção de frutos. A seleção do melhor porta-enxerto conduz a uma média da população melhorada de 75,48 , produzindo um ganho genético de $16,3 \%$ na produção de frutos. Esse ganho é expressivo e deverá contribuir muito para o setor produtivo.

Para a altura da planta em 2010, a estimativa da herdabilidade individual no sentido amplo foi de $77,97 \%$. Essa herdabilidade é alta e reflete, provavelmente, um caráter quantitativo governado por poucos genes e menor influência ambiental. Esses resultados concordam com aqueles obtidos para a altura de cajueiros, conforme relatado por Cavalcanti et al. (2007). Nesse caso, a seleção, para ser precisa, não necessitaria da coleta de dados em várias safras em cada genótipo. A herdabilidade ao nível de médias de cinco plantas de um mesmo genótipo, em uma safra, foi de $91,39 \%$. Esse valor é ótimo para a seleção, pois conduz a uma acurácia seletiva de 95,60\% (Tabela 3).

Para a eficiência produtiva na média de quatro safras, a estimativa da herdabilidade da média das cinco plantas em quatro safras foi de 59,93\%. Esse valor é ótimo para a seleção, pois conduz a uma acurácia seletiva de 77,42\% (Tabela 3). Assim, seleções eficientes podem ser realizadas para cada uma das três variáveis.

Verificou-se correlação alta e positiva $(\mathrm{r}=$ 0,7164 ) entre a altura da planta e a produção de frutos. Ou seja, quanto mais alta a planta, maior a produção de frutos. Assim, a seleção simultânea para alta produção e porte reduzido deve basear-se na variável eficiência produtiva, que considera a produção de frutos por volume de copa. Essa apresenta correlações negativas tanto com a produção de frutos $(\mathrm{r}=-0,2925)$ quanto com a altura da planta $(\mathrm{r}=-0,7589)$, porém de baixa magnitude com a produção de frutos.

As médias genéticas preditas para o caráter eficiência produtiva, bem como seus limites inferiores (LIIC) e superiores (LSIC) do intervalo de confiança a 95\% de confiança, são apresentadas na Tabela 5.Verifica-se que, dentre os 10 melhores genótipos para produção de frutos (Tabela 4) e para eficiência produtiva (Tabela 5), apenas três (1.522; 868 e 880) são coincidentes. Isso é reflexo da correlação negativa e baixa entre as duas variáveis. Assim, como passo seguinte do melhoramento, indica-se o cruzamento entre os melhores genótipos das Tabelas 4 e 5, com vistas à obtenção de indivíduos recombinantes, congregando alta produtividade de frutos e baixo porte.

A Tabela 6 apresenta o agrupamento multivariado dos genótipos pelo método de Tocher aplicado sobre as distâncias genéticas euclidianas médias (RESENDE, 2007). Verificou-se a formação de seis grupos diferentes quanto à divergência genética entre os porta-enxertos. Os cinco genótipos superiores para a eficiência produtiva encontram-se nos grupos 2 e 6 , e para a produção de frutos encontram-se no grupo 1. Assim, o cruzamento entre os melhores genótipos para essas duas variáveis, além de conduzir à obtenção de indivíduos recombinantes, congregando alta produtividade de frutos e baixo porte, deverá produzir heterose para a produção de frutos, caráter esse que geralmente apresenta dominância alélica. 
TABELA 1 - Número de registro, nomes comum e científico no Banco Ativo de Germoplasma de Citros do Centro APTA Citros Sylvio Moreira, dos genótipos dos limões Cravo, Volkameriano e Rugoso.

\begin{tabular}{|c|c|c|}
\hline $\begin{array}{l}\text { Número de } \\
\text { registro }\end{array}$ & Nome comum & Nome científico \\
\hline 374 & Limão- Volkameriano- Catania 2 & C. volkameriana \\
\hline 859 & Rangpur -Otaheite -Orange 12901 & C. limonia \\
\hline 860 & Rangpur- India 26.1 & C. limonia \\
\hline 861 & Rangpur- Red- Ling -Ming 93377 & C. limonia \\
\hline 862 & Rangpur -Red -Lime D33.40 & C. limonia \\
\hline 863 & Limão- Cravo -Limeira & C. limonia \\
\hline 864 & Rangpur- Rose -Lemon 124684 & C. limonia \\
\hline 865 & Rangpur- Kusaie -Lime & C. limonia \\
\hline 866 & Rangpur-Red- Lime D33.30 & C. limonia \\
\hline 867 & Rangpur- Red -Lime D33.47 & C. limonia \\
\hline 868 & Rangpur- Rose- Lime & C. limonia \\
\hline 869 & Limão- Cravo- Taquaritinga & C. limonia \\
\hline 871 & Limão- Cravo- EEL & C. limonia \\
\hline 872 & Rangpur -Egyptian-Lime & C. limonia \\
\hline 873 & Rangpur- Lemon-India & C. limonia \\
\hline 874 & Rangpur-Borneo- Red & C. limonia \\
\hline 875 & Rangpur -Australian -Red & C. limonia \\
\hline 880 & Citrus pennivesiculata & C. pennivesiculata \\
\hline 882 & Japanshe Citroen & C. limonia \\
\hline 883 & Phillipine- Red- Lime & C. limonia \\
\hline 884 & Santa -Bárbara -Red -Lime & C. limonia \\
\hline 1.467 & Limão- Cravo- da- Califórnia & C. limonia \\
\hline 1.468 & Limão -Cravo x Laranja- Azeda & C. limonia $x$ C. aurantium \\
\hline 1.522 & Limão -Cravo -Ipanema & C. limonia \\
\hline 1.524 & Limão- Cravo x Citrange -Carrizo & C. limonia $x$ (P.trifoliata $x$ C. sinensis) \\
\hline 1.538 & Limão -Rugoso FM & C. jambhiri \\
\hline 1.539 & Limão -Rugoso Schaub & C. jambhiri \\
\hline 1.642 & Rangpur- Lime x Trifoliata 60110 & C. limonia $x$ P. trifoliata \\
\hline 1.643 & Limão- Rugoso -Do Cabo & C. jambhiri \\
\hline 1.644 & Rangpur -Lime x Trifoliata 5320 & C. limonia x P.trifoliata \\
\hline 1.648 & Rangpur- Lime x Trifoliata 3810 & C. limonia $\times$ P. trifoliata \\
\hline 1.649 & Citrus kokhai & C. kokhai \\
\hline 1.655 & Limão- Rugoso 58329 & C. jambhiri \\
\hline 1.659 & Limão-Rugoso 58328 & C. jambhiri \\
\hline 1.695 & Limão- Cravo x Trifoliata Swingle B & C. limonia $x$ P. trifoliata \\
\hline 1.707 & Limão -Cravo x Trifoliata Swingle A & C. limonia $x$ P. trifoliata \\
\hline
\end{tabular}


TABELA 2 - Produções médias anuais de frutos, eficiências produtivas médias anuais e altura média no sétimo ano de laranjeiras Valência enxertadas em seleções e híbridos dos limões Cravo, Volkameriano e Rugoso. Comendador Gomes-MG.

\begin{tabular}{|c|c|c|c|c|c|c|c|c|c|c|c|c|}
\hline \multirow[t]{2}{*}{ Genótipo } & \multirow{2}{*}{2006} & \multicolumn{5}{|c|}{ Produção } & \multicolumn{5}{|c|}{ Eficiência produtiva } & \multirow{2}{*}{$\frac{\text { Altura }}{2010}$} \\
\hline & & 2007 & 2008 & 2009 & 2010 & Media & 2007 & 2008 & 2009 & 2010 & Media & \\
\hline & & & & g.pl-1 & & & & & kg.m- & & & $\mathrm{m}$ \\
\hline 374 & 50,2 & 67,6 & 68,2 & 44,7 & 114,2 & 69,0 & 11,9 & 7,3 & 3,0 & 7,0 & 7,3 & 3,0 \\
\hline 860 & 25,3 & 51,2 & 59,0 & 90,6 & 127,8 & 70,8 & 7,6 & 5,7 & 5,5 & 7,1 & 6,5 & 3,1 \\
\hline 861 & 23,1 & 52,9 & 83,3 & 77,8 & 122,4 & 71,9 & 7,0 & 8,6 & 5,1 & 7,5 & 7,1 & 3,1 \\
\hline 862 & 20,7 & 57,8 & 65,7 & 76,7 & 123,0 & 68,8 & 8,8 & 8,1 & 5,0 & 8,4 & 7,6 & 3,0 \\
\hline 863 & 45,4 & 85,5 & 60,3 & 52,2 & 138,6 & 76,4 & 11,5 & 5,5 & 3,1 & 7,7 & 7,0 & 3,3 \\
\hline 864 & 29,0 & 64,0 & 60,6 & 49,7 & 112,8 & 63,2 & 13,1 & 8,4 & 4,1 & 8,0 & 8,4 & 3,0 \\
\hline 865 & 44,2 & 67,8 & 35,8 & 43,2 & 106,6 & 59,5 & 12,2 & 5,2 & 2,8 & 6,0 & 6,6 & 3,0 \\
\hline 867 & 33,0 & 58,0 & 49,2 & 48,3 & 88,4 & 55,4 & 13,7 & 8,0 & 4,6 & 7,3 & 8,4 & 2,8 \\
\hline 868 & 39,8 & 69,2 & 49,0 & 79,8 & 124,4 & 72,4 & 12,2 & 6,0 & 5,3 & 8,2 & 7,9 & 3,0 \\
\hline 869 & 33,0 & 73,3 & 67,7 & 71,2 & 131,2 & 75,3 & 10,7 & 6,7 & 5,4 & 8,5 & 7,9 & 3,1 \\
\hline 872 & 42,5 & 72,0 & 42,8 & 69,6 & 89,1 & 63,2 & 14,7 & 5,3 & 4,6 & 5,5 & 7,6 & 3,0 \\
\hline 873 & 44,1 & 33,2 & 70,4 & 10,6 & 94,6 & 50,6 & 4,7 & 9,0 & 0,8 & 6,9 & 5,3 & 2,9 \\
\hline 875 & 44,0 & 63,2 & 60,8 & 60,9 & 94,4 & 64,7 & 13,0 & 6,7 & 5,2 & 6,6 & 7,9 & 2,8 \\
\hline 880 & 37,6 & 78,3 & 69,6 & 79,5 & 88,8 & 70,8 & 16,9 & 11,2 & 6,7 & 6,3 & 10,3 & 3,0 \\
\hline 882 & 27,2 & 61,0 & 38,6 & 29,9 & 96,0 & 50,6 & 14,6 & 6,4 & 2,5 & 8,2 & 7,9 & 2,7 \\
\hline 883 & 32,2 & 59,1 & 72,3 & 72,2 & 103,4 & 67,8 & 9,1 & 8,2 & 5,3 & 6,7 & 7,3 & 3,0 \\
\hline 884 & 39,7 & 51,8 & 82,5 & 65,2 & 147,8 & 77,4 & 8,5 & 9,7 & 4,5 & 8,8 & 7,9 & 3,1 \\
\hline 1.467 & 35,6 & 64,9 & 64,3 & 66,7 & 129,2 & 72,1 & 10,1 & 5,9 & 4,7 & 7,6 & 7,1 & 3,1 \\
\hline 1.468 & 29,2 & 55,9 & 62,6 & 70,1 & 121,6 & 67,9 & 8,4 & 5,9 & 4,8 & 7,5 & 6,7 & 3,1 \\
\hline 1.522 & 39,8 & 60,0 & 87,6 & 60,8 & 150,2 & 79,7 & 9,2 & 10,3 & 4,3 & 9,3 & 8,3 & 3,1 \\
\hline 1.524 & 28,0 & 65,2 & 40,9 & 56,5 & 103,8 & 58,9 & 18,3 & 7,3 & 5,8 & 10,1 & 10,4 & 2,2 \\
\hline 1.538 & 47,4 & 55,2 & 37,5 & 48,4 & 113,8 & 60,5 & 9,4 & 4,1 & 3,1 & 7,3 & 6,0 & 3,0 \\
\hline 1.539 & 44,4 & 73,8 & 22,0 & 47,5 & 133,6 & 64,3 & 10,7 & 1,8 & 2,6 & 7,3 & 5,6 & 3,2 \\
\hline 1.642 & 8,2 & 77,6 & 28,4 & 73,5 & 96,0 & 56,7 & 16,0 & 3,2 & 4,8 & 6,6 & 7,7 & 3,0 \\
\hline 1.643 & 43,7 & 65,7 & 39,4 & 99,4 & 124,2 & 74,5 & 11,1 & 3,7 & 6,0 & 7,6 & 7,1 & 3,1 \\
\hline 1.644 & 18,1 & 51,1 & 47,6 & 47,9 & 91,6 & 51,3 & 16,8 & 9,4 & 6,5 & 11,4 & 11,0 & 2,3 \\
\hline 1.648 & 19,3 & 59,1 & 38,4 & 53,2 & 94,4 & 52,9 & 17,7 & 8,3 & 6,8 & 13,1 & 11,4 & 2,2 \\
\hline 1.659 & 55,6 & 56,6 & 26,7 & 51,8 & 109,4 & 60,0 & 10,1 & 3,4 & 4,0 & 7,5 & 6,2 & 2,8 \\
\hline 1.707 & 17,9 & 47,7 & 25,3 & 44,8 & 101,8 & 47,5 & 16,8 & 5,8 & 5,5 & 11,5 & 9,9 & 2,5 \\
\hline
\end{tabular}


TABELA 3 - Estimativas de parâmetros genéticos para a produção de frutos, altura da planta e eficiência produtiva de laranjeiras Valência enxertadas em limoeiros Cravo, Volkameriano e Rugoso. Comendador Gomes-MG.

\begin{tabular}{lccc}
\hline \multirow{2}{*}{\multicolumn{1}{c}{ Parâmetros genéticos }} & \multicolumn{3}{c}{ Estimativas } \\
\cline { 2 - 4 } & $\begin{array}{c}\text { Produção } \\
\text { (média de 5 safras) }\end{array}$ & $\begin{array}{c}\text { Altura } \\
\text { (em 2010) }\end{array}$ & $\begin{array}{c}\text { Eficiência Produtiva } \\
\text { (média de 4 safras) }\end{array}$ \\
\hline Variância genotípica & 55,43 & 0,07 & 1,27 \\
Variância de ambiente permanente & 2,75 & - & - \\
Variância residual & 536,66 & 0,02 & 4,26 \\
Variância fenotípica individual & 594,85 & 0,09 & 5,54 \\
Herdabilidade individual no sentido amplo & $0,093 \pm 0,03$ & 0,77 & - \\
Herdabilidade ao nível de médias de cinco plantas em & 0,33 & 0,91 & 0,23 \\
uma safra & & & 0,60 \\
Herdabilidade ao nível de médias de cinco plantas & 0,71 & - & - \\
nas safras & $0,097 \pm 0,03$ & - & - \\
Repetibilidade individual & 0,35 & - & \\
Repetibilidade ao nível de médias de cinco safras & 0,58 & 0,95 & - \\
Acurácia na seleção de genótipos em uma safra & 0,84 & - & 7,79 \\
Acurácia na seleção de genótipos nas safras & 64,91 & 2,91 & \\
Média geral & & & \\
\hline
\end{tabular}

TABELA 4 - Médias genéticas preditas para o caráter produção de frutos, bem como seus limites inferiores (LIIC) e superiores (LSIC) do intervalo de confiança.

\begin{tabular}{|c|c|c|c|c|c|}
\hline Ordem & Genótipo & Média Genética & LIIC & LSIC & Média Fenotípica \\
\hline 1 & 1522 & 75,48 & 67,37 & 83,59 & 79,67 \\
\hline 2 & 884 & 73,85 & 65,74 & 81,97 & 77,40 \\
\hline 3 & 863 & 73,12 & 65,01 & 81,23 & 76,38 \\
\hline 4 & 869 & 72,34 & 64,23 & 80,46 & 75,29 \\
\hline 5 & 1643 & 71,75 & 63,64 & 79,86 & 74,46 \\
\hline 6 & 868 & 70,31 & 62,20 & 78,42 & 72,45 \\
\hline 7 & 1.467 & 70,09 & 61,98 & 78,20 & 72,15 \\
\hline 8 & 861 & 69,91 & 61,80 & 78,02 & 71,89 \\
\hline 9 & 860 & 69,11 & 61,00 & 77,22 & 70,78 \\
\hline 10 & 880 & 69,11 & 60,99 & 77,22 & 70,77 \\
\hline 11 & 374 & 67,83 & 59,71 & 75,94 & 68,98 \\
\hline 12 & 862 & 67,69 & 59,57 & 75,80 & 68,78 \\
\hline 13 & 1.468 & 67,03 & 58,92 & 75,14 & 67,87 \\
\hline 14 & 883 & 67,00 & 58,89 & 75,12 & 67,83 \\
\hline 15 & 875 & 64,74 & 56,63 & 72,85 & 64,67 \\
\hline 16 & 1.539 & 64,47 & 56,36 & 72,58 & 64,29 \\
\hline 17 & 865 & 64,31 & 56,10 & 72,52 & 62,02 \\
\hline 18 & 864 & 63,72 & 55,61 & 71,84 & 63,25 \\
\hline 19 & 872 & 63,68 & 55,57 & 71,79 & 63,19 \\
\hline 20 & 1.538 & 61,73 & 53,61 & 69,84 & 60,46 \\
\hline 21 & 1.659 & 61,40 & 53,29 & 69,52 & 60,01 \\
\hline 22 & 1.524 & 60,59 & 52,48 & 68,71 & 58,88 \\
\hline 23 & 1.642 & 59,06 & 50,95 & 67,17 & 56,73 \\
\hline 24 & 867 & 58,08 & 49,97 & 66,20 & 55,37 \\
\hline 25 & 882 & 57,70 & 49,38 & 66,02 & 54,95 \\
\hline 26 & 1.648 & 56,31 & 48,20 & 64,43 & 52,90 \\
\hline 27 & 1.644 & 55,14 & 47,03 & 63,25 & 51,26 \\
\hline 28 & 873 & 54,66 & 46,55 & 62,77 & 50,59 \\
\hline 29 & 1.707 & 52,45 & 44,34 & 60,56 & 47,50 \\
\hline
\end{tabular}


TABELA 5 - Médias genéticas preditas para o caráter eficiência produtiva, bem como seus limites inferiores (LIIC) e superiores (LSIC) do intervalo de confiança.

\begin{tabular}{|c|c|c|c|c|c|}
\hline Ordem & Genótipo & Média Genética & LIIC & LSIC & Média Fenotípica \\
\hline 1 & 1.648 & 9,78 & 8,26 & 11,30 & 11,44 \\
\hline 2 & 1.644 & 9,55 & 8,03 & 11,07 & 11,02 \\
\hline 3 & 1.524 & 9,20 & 7,67 & 10,72 & 10,37 \\
\hline 4 & 880 & 9,14 & 7,62 & 10,67 & 10,27 \\
\hline 5 & 1.707 & 8,94 & 7,41 & 10,46 & 9,89 \\
\hline 6 & 864 & 8,13 & 6,61 & 9,65 & 8,41 \\
\hline 7 & 867 & 8,13 & 6,60 & 9,65 & 8,41 \\
\hline 8 & 1.522 & 8,05 & 6,53 & 9,57 & 8,26 \\
\hline 9 & 868 & 7,87 & 6,35 & 9,40 & 7,94 \\
\hline 10 & 882 & 7,86 & 6,34 & 9,39 & 7,92 \\
\hline 11 & 875 & 7,85 & 6,32 & 9,37 & 7,89 \\
\hline 12 & 884 & 7,85 & 6,32 & 9,37 & 7,89 \\
\hline 13 & 869 & 7,83 & 6,31 & 9,35 & 7,86 \\
\hline 14 & 1.642 & 7,72 & 6,19 & 9,24 & 7,65 \\
\hline 15 & 862 & 7,70 & 6,17 & 9,22 & 7,61 \\
\hline 16 & 872 & 7,66 & 6,14 & 9,19 & 7,55 \\
\hline 17 & 374 & 7,53 & 6,00 & 9,05 & 7,30 \\
\hline 18 & 883 & 7,52 & 6,00 & 9,05 & 7,29 \\
\hline 19 & 1.467 & 7,42 & 5,89 & 8,94 & 7,10 \\
\hline 20 & 1.643 & 7,41 & 5,89 & 8,94 & 7,09 \\
\hline 21 & 861 & 7,40 & 5,87 & 8,92 & 7,06 \\
\hline 22 & 863 & 7,35 & 5,82 & 8,87 & 6,97 \\
\hline 23 & 1.468 & 7,18 & 5,65 & 8,70 & 6,66 \\
\hline 24 & 865 & 7,14 & 5,62 & 8,66 & 6,59 \\
\hline 25 & 860 & 7,07 & 5,55 & 8,60 & 6,47 \\
\hline 26 & 1.659 & 6,95 & 5,42 & 8,47 & 6,24 \\
\hline 27 & 1.538 & 6,81 & 5,28 & 8,33 & 5,98 \\
\hline 28 & 1.539 & 6,61 & 5,09 & 8,14 & 5,62 \\
\hline 29 & 873 & 6,45 & 4,93 & 7,98 & 5,33 \\
\hline
\end{tabular}

TABELA 6 - Agrupamento multivariado dos genótipos pelo método de Tocher aplicado sobre as distâncias genéticas euclidianas médias.

\begin{tabular}{cl}
\hline Grupos & \multicolumn{1}{c}{ Genótipos } \\
\hline $\mathbf{1}$ & $863,869,1.467,1.468,884,1.522,860,861,862,864,865,868,872,875,374,1.643,883$ \\
$\mathbf{2}$ & $1.524,1.707,1.648,1.644$ \\
$\mathbf{3}$ & $867,1.659,1.538,882,1.642$ \\
$\mathbf{4}$ & 873 \\
$\mathbf{5}$ & 1.539 \\
$\mathbf{6}$ & 880 \\
\hline
\end{tabular}




\section{CONCLUSÕES}

1-Para o parâmetro produção de frutos, a acurácia seletiva de cinco colheitas é de $84,59 \%$. Ganhos genéticos de 11,5\% na produção de frutos são obtidos pela seleção dos sete melhores porta-enxertos, enquanto a seleção do melhor genótipo confere ganho genético de $16,3 \%$.

2-A acurácia seletiva do parâmetro eficiência produtiva, para quatro colheitas, é $77,4 \%$.

3-As melhores médias genéticas preditas $\left(>70,0 \mathrm{~kg} \cdot \mathrm{pl}^{-1}\right)$ para o caráter produção de frutos são obtidas pelos genótipos Limão-Cravo- Ipanema (1522), Santa-Bárbara- Red -Lime (884), LimãoCravo- Limeira (863), Limão- Cravo- Taquaritinga (869), Limão- Rugoso -do -Cabo (1643), RangpurRose -Lime (868) e Limão- Cravo- da- Califórnia (1467).

4-Para o parâmetro eficiência produtiva, as maiores médias genéticas $\left(>8,0 \mathrm{~kg} \cdot \mathrm{m}^{-3}\right)$ são para os genótipos Rangpur-Lime x Trifoliata 3810 (1648), Rangpur- Lime x Trifoliata 5320 (1644), Limão-Cravo x Citrange- Carrizo (1524), Citrus pennivesiculata (880), Limão- Cravo x Trifoliata- Swingle A (1707), Rangpur- Rose -Lemon 124684 (864), Rangpur- Red- Lime D33.47 (867) e Limão Cravo Ipanema (1522).

5-Dentre os 10 melhores genótipos para produção de frutos e para eficiência produtiva, apenas três são coincidentes: Rangpur- Rose- Lime (868), Citrus pennivesiculata (880) e Limão- Cravo- Ipanema (1522).

\section{AGRADECIMENTOS}

Ao Conselho Nacional de Desenvolvimento Científico e Tecnológico, à Fundação de Amparo à Pesquisa do Estado de São Paulo e ao Fundo de Defesa da Citricultura, pelo suporte financeiro. Ao United States Horticultural Research Laboratory, Florida, EUA, pela cessão de parte dos porta-enxertos. À Sucocítrico Cutrale S/A, especialmente aos Eng. Agr. Luiz Fernando Girotto e Antônio Ricardo Violante, pela área e manutenção do experimento.

\section{REFERÊNCIAS}

BASSANEZI, R.B.; BERGAMIN FILHO, A.; AMORIM, L.; GIMENES-FERNANDES, N.; GOTTWALD, T.R.; BOVE, J.M. Spatial and temporal analyses of citrus sudden death as a tool to generate hypotheses concerning its etiology. Phytopathology, Saint Paul, v.93, p. 502-512, 2003.

CAVALCANTI, J. J. V.; RESENDE, M. D. V. de; CRISÓSTOMO, J. R.; BARROS, L. M.; PAIVA, J. R. de. Genetic control of quantitative traits and hybrid breeding strategies for Cashew improvement. Crop Breeding and Applied Biotechnology, Londrina, v. 7, p. 186-195, 2007.

MACCHERONI, W.; ALEGRIA, M.C.; GREGGIO, C.C.; PIAZZA, J.P;, KAMLA, R.F; ZACHARIAS, P.R.; BAR-JOSEPH, M.; KITAJIMA, E.W.; ASSUMPÇÃO, L.C.; CAMAROTTE, G.; CARDOZO, J.; CASAGRANDE, E.C.; FERRARI, F.; FRANCO, S.F.; GIACHETTO, P.F.; GIRASOL, A.; JORDAO, H.; SILVA, V.H.; SOUZA, L.C.; AGUILAR-VILDOSO, C.I.; ZANCA, A.S.; ARRUDA, P.; KITAJIMA, J.P.; REINACH, F.C.; FERRO, J.A.; DA SILVA, A.C. Identification and genomic characterization of a new virus (Tymoviridae family) associated with citrus sudden death disease. Journal of Virology, Washington, v.79, p.3028-3037, 2005.

MENDEL, K. Rootstock-scion relationships in Shamouti trees on light soils. Katavim, Rehovot, v.6, p. 35-60, 1956.

POMPEU JUNIOR, J.; BLUMER, S. Híbridos de trifoliata como porta-enxertos para a laranjeira Valência. Pesquisa Agropecuária Brasileira, Brasília, v.44, n.7, p.701-705, 2009.

POMPEU JUNIOR, J. ; BLUMER, S . Incompatibilidade da laranjeira Valência enxertada em limão Cravo x citrange Carrizo. In: SEMINÁRIO INTERNACIONAL DE CITROS:MELHORAMENTO, 7., 2002, Bebedouro. Anais... Bebedouro: Estação Experimental de Citricultura de Bebedouro, 2002. p.126.

POMPEU JUNIOR, J.; BLUMER, S. Morte súbita dos citros: suscetibilidade de seleções de limão cravo e uso de interenxertos. Revista Brasileira de Fruticultura, Jaboticabal, v. 30, p. 1159-1161, 2008. 
RESENDE, M. D. V. Genética biométrica e estatística no melhoramento de plantas perenes. Brasília: Embrapa Informação Tecnológica, 2002. 975p.

RESENDE, M. D. V. Matemática e estatística na análise de experimentos e no melhoramento genético. Colombo: Embrapa Florestas, 2007. 561p.

RESENDE, M. D. V.; DUARTE, J. B. Precisão e controle de qualidade em experimentos de avaliação de cultivares. Pesquisa Agropecuária Tropical, Goiânia, v. 37, n. 3, p. 182-194, 2007.

ROMAN, M. P.; CAMBRA, M.; JUAREZ, J.; MORENO, P.; VILA, N. D.; TANAKA, F.A.O.;ALVES, E.; KITAJIMA, E.W.; YAMAMOTO, P.T.; BASSANEZI, R.B.; TEIXEIRA, D.C.; JESUS JUNIOR, W.C.; AYRES, A.J.; FERNANDES, N.G.; RABENSTEIN, F.; BOVÉ, J.M. Sudden death of citrus in Brazil: a graft-transmissible bud union disease. Plant Disease, Saint Paul, v.88, p.453-467, 2004.
SETZER, J. Atlas climático e ecológico do Estado de São Paulo. São Paulo: CIBPU, 1966. 61p.

YAMAMOTO, P. T.; BASSANEZI, R. B.; WULFF, N. A.; SANTOS, M. A.; SANCHES, A. L.; TOLOY, R. S.; GIMENES-FERNANDES, N.; AYRES, A. J.; JESUS JUNIOR, W.C.; NAGATA, T.; TANAKA, F. A. O.; KITAJIMA, E. W. ; BOVÉ, J. M. Citrus sudden death is transmitted by graft-inoculation and natural transmission is prevented by individual insect-proof cages. Plant Disease, Saint Paul, v. 95, p. 104-112, 2011. 